\title{
Error Analysis of a Low Cost TDoA Sensor Network
}

\author{
Noha El Gemayel, Holger Jäkel and Friedrich K. Jondral \\ Communications Engineering Lab, Karlsruhe Institute of Technology (KIT), Germany \\ \{noha.gemayel, holger.jaekel, friedrich.jondral\}@kit.edu
}

\begin{abstract}
Geolocation methods are recently gaining a lot of interest due to their new range of applicability. Location based mobile services as well as frequency regulators aiming at efficient spectrum usage are interested in flexible, low cost geolocation systems with high accuracy. One method that meets those requirements is Time Difference of Arrival (TDoA). It has been subject to research for many years now. The main focus of research published about TDoA is presenting new algorithms or calculating estimation bounds in different scenarios. Due to new low cost available hardware solutions, a simple TDoA system can be built and used as a testbed for different algorithms in different real scenarios. This contribution presents an error analysis of a TDoA sensor network using low cost, off-the-shelf software defined radio platforms. The system relies on GPS time stamps provided by the platforms. Five important TDoA error types caused by hardware as well as different channel effects are analyzed. Each error is analyzed in its influence on the position estimate and a possible solution is given. Finally, a general structure of how a smart TDoA system should work is described.
\end{abstract}

\section{INTRODUCTION}

Time Difference of Arrival (TDoA) presents a good solution for applications requiring passive localization. Compared to other methods, TDoA offers a low cost solution with accurate results. Passive localization can be applied in security and emergency cases, as well as by frequency regulators in a frequency monitoring and management system aiming at efficient spectrum usage. These tasks require a localization system with a wide range of applicability and high accuracy.

A large number of algorithms and methods presented throughout the years have dealt with specific TDoA challenges. Estimating the TDoAs from received signals can be a problem due to low signal-to-noise ratio (SNR), multipath propagation, non-line-of-sight (NLOS) propagation and time and frequency synchronization errors. Algorithms for estimating the relative time delay vary from simple or prefiltered correlation methods [1] to super resolution algorithms [2] or maximum likelihood-based algorithms [3], [4] that can be used in multipath scenarios. Localization algorithms try to overcome the resulting errors of TDoA estimates. When dealing with additive random errors, minimizing the noise can be done by least squares estimation [5], [6] or by Kalman Filters for nonlinear systems. On the other hand, mitigating resulting bias from NLOS errors can be done by eliminating NLOS sensors after identifying them [7] or by weighting the sensors' estimates according to their reliability [8]. These algorithms present good solutions to a number of problems.
The question remains on whether and when they can be applied in real scenarios.

The software radio platforms provided by Ettus [9] present a testbed that can be used to verify these algorithms in different real scenarios. This paper presents an error analysis of TDoA measurements obtained by using Ettus USRPs as TDoA sensors and relates the results to methods previously described in the literature, thereby validating their applicability. The implemented algorithms are combined in an intelligent TDoA system that can be applied in a wide range of scenarios.

The paper is organized as follows. Section II describes the setup of the sensors, the transmitter, the transmitted signal as well as the TDoA system. Section III analyzes five different error types that can be seen from the measurement. Section IV describes the effect of the presented errors on the resulting position estimate and discusses methods to overcome these challenges. Section V concludes the paper and presents ideas for further work.

\section{Measurement Setup}

The measurement consists of six identical sensor stations which are installed on the rooftops of suitable campus buildings as can be seen in Fig. 1. Each sensor containts a Universal Software Radio Peripheral 2 (USRP2) with a WBX daugterboard [9]. This enables the sensors to record IQ-data in a wide spectrum range $(50 \mathrm{MHz}-2.2 \mathrm{GHz})$ using a wide range of sample rates (up to $25 \mathrm{MHz}$ ). The USRP is conntected to and controlled by a PC with a large storage space. The sampled IQ-data is time stamped using a GPS module which provides high accurate time measurements and is also used to discipline the USRPs oscillators.

The transmitter to be localized sends a signal also using a USRP2, an antenna and an amplifier. The transmitted signal consists of $2 \mathrm{MHz}$ of band limited white noise, from which $1 \mathrm{MHz}$ is filtered at the receiver and processed for the TDoA estimation. The center frequency was set to $431 \mathrm{MHz}$.

The sensors' sample rate was set to $5 \mathrm{MHz}$. After gathering the IQ-data from all sensors, the band of interest is filtered using a lowpass filter. Afterwards, one sensor is chosen as reference sensor (here: sensor 1). The simplest relative time delay estimation method is to cross-correlate received signals from the other five sensors with the received signal of the reference sensor. The resulting correlation should peak at the true time delay. To enhance accuracy, a quadratic interpolation is carried out around the detected peak. Localization algorithms 




Fig. 1. Measurement Setup at the University Campus

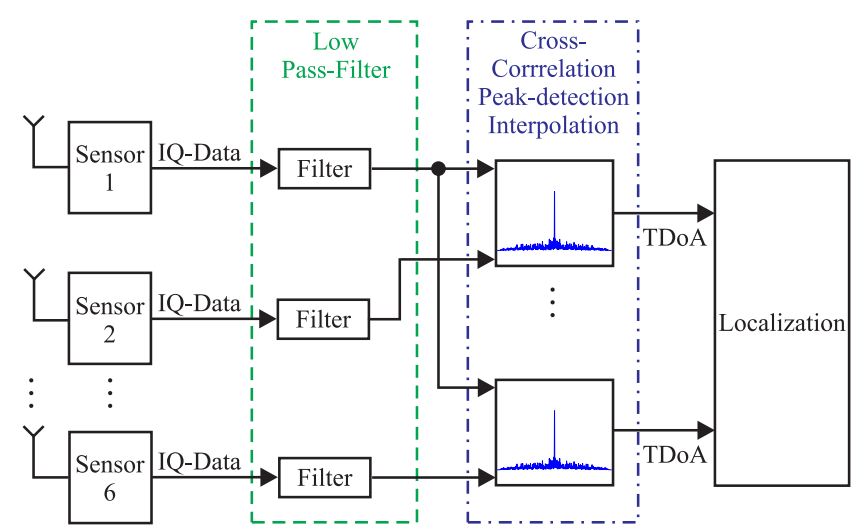

Fig. 2. TDoA system setup

transform the estimated time differences into delay differences and solve the resulting hyperbolic equation system to obtain a position estimate. Fig. 2 gives an overview of the TDoA system setup.

The error analysis is based on time delay estimation errors and their effect on the position estimate. The true position was logged using an accurate GPS receiver. For identifying different error sources, two measurement scenarios are depicted: one where the transmitter is static, and one where it simulates a pedestrian (Fig. 1). These scenarios help identify five distinct types of TDoA measurement errors that will be presented in the next section.

\section{Measurement ERrors}

The static scenario is studied first. The difference between good and bad channels, depending on the distance as well as the buidings around the sensors, can be seen in the spectra in Fig. 3. Sensor 1 is only $86 \mathrm{~m}$ away and is receiving a strong and direct path from the transmitter. Sensor 2 is far and is covered by a complete building and sensor 6 is further and is surrounded by many buildings. The sensors also receive other signals at about $-2 \mathrm{MHz}$, which in that case are private mobile radio signals.

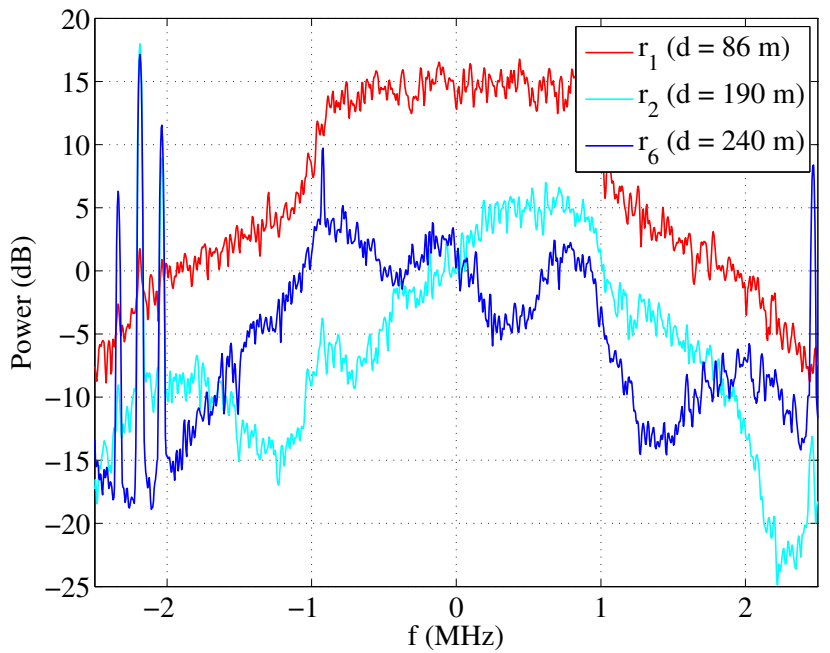

Fig. 3. Received Spectra of Signals



Fig. 4. TDoA Error type 1

For a first look at the system errors, the TDoA output after the correlation, peak detection and interpolation block (Fig. 2) is compared to the true delay. The estimation error at measurement $n$ using sensor $i$ and sensor 1 (reference sensor) is calculated by:

$$
e(n)=c \cdot\left(\hat{\tau}_{i, 1}-\tau_{i, 1}\right)
$$

where $\tau_{i, 1}$ is the true delay, $\hat{\tau}_{i, j}$ is the estimated delay and $c$ is the speed of light. The observation time for each correlation is $1 \mathrm{~ms}$ and the update rate is $0.1 \mathrm{~s}$. Figure 4 shows the errors of the TDoA estimates over 50 measurements ( 5 seconds).

\section{A. Error source 1: Insufficient SNR}

The first error that can be seen in Fig. 4 is a large, random error at two sensors $\left(\tau_{2,1}, \tau_{6,1}\right)$ marked with $(\square)$ and $(\triangle)$. The error shows that those TDoA estimates are random. This can be explained by looking at the cross-correlation in Fig. 5. It is obvious that the received signal has either too low SNR 


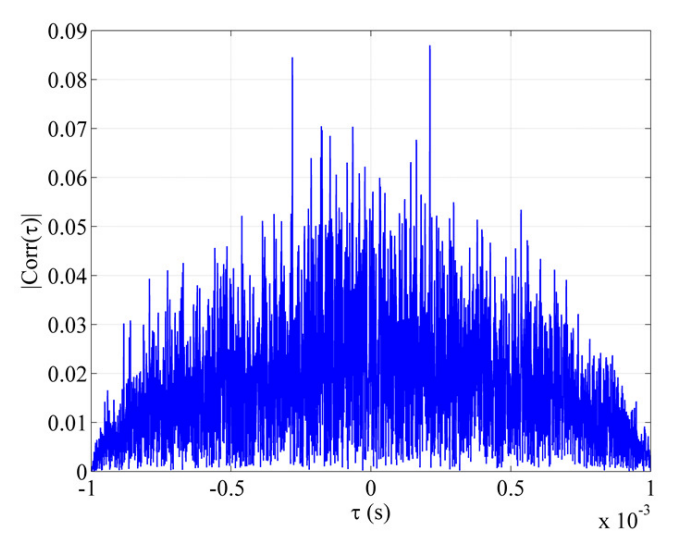

Fig. 5. Cross-correlation with a signal with too low SNR



Fig. 6. TDoA Error type 2, 3 and 4

or is not the transmitted signal. The cross-correlation shows the absence of a clear correlation peak which results in this error. Detecting the error can be easily done by measuring the peak of the normalized cross-correlation. Looking at larger observation windows of the signals can sometimes lead to a clear peak. In this case, this did not help. Later, the results will show how TDoA estimates with no clear correlation peak should not be used for further calculation of the position. For observing the other errors, TDoA estimates of sensors 2 and 6 have been removed in Fig. 6.

\section{B. Measurement noise}

Depending mainly on SNR and observation time, estimated time delays vary around a specific value. This error can be observed by looking at the variation of the estimates over time (Fig. 6, Error type 2). It can be compensated by either looking at longer observation windows or averaging over a number of estimates. Here, the errors have the standard deviations of $\sigma_{3,1}=12 \mathrm{~m}, \sigma_{4,1}=7 \mathrm{~m}$ and $\sigma_{5,1}=7 \mathrm{~m}$.

\section{Synchronization errors}

Bias due to GPS time error: Time synchronization among the sensors is done using GPS. The used hardware gives a specific accuracy (here: 1PPS-accuracy of $15 \mathrm{~ns}$ ). In the worst case, synchronization errors of two sensors will add up in the correlation operation. This error can be seen in the error average in Fig. 6 (Error type 3), here the errors are $13 \mathrm{~m}$ and $6.5 \mathrm{~m}$.

\section{Non-Line-of-Sight propagation}

Bias due to NLOS: Non-line-of-sight is by far the biggest challenge for TDoA. This error makes the correlation peak appear at a false time delay and leads to biased estimates. Here, NLOS can be seen in the average of $\tau_{3,1}$ (Fig. 6, Error type 4). Its value is around $65 \mathrm{~m}$. Detecting this error is a tough task that was discussed in a number of papers [8], [7]. Many methods try to identify NLOS-sensors by assuming a higher measurement noise of these sensors [7]. The detected NLOS sensors can either be eliminated or weighted according to their reliability. An alternative method would be to define subsets of the sensors, calculate a position estimate as well as a residual for each subset, and weight the position estimates accordingly [8]. This method needs subsets of at least 4 sensors to be able to calulate their residuals [10].

\section{E. Multipath propagation}

Bias due to multipath: In dense multipath scenarios, multiple correlation peaks resulting from the sum of delayed versions of the signal can overlap. This happens mainly with narrow band signals that have broader correlation peaks in time. The overlapping of two peaks leads to new constellations, with broader peaks at wrong delays. This error can be detected by observing the peak width of the autocorrelations of the received signals. Here, we depicted a scenario where sensor 3 had a dense multipath channel. Fig. 7 shows the autocorrelation of the received signals of sensor 1 and 3. Sensor 1 shows the expected shape of the autocorrelation, considering the signal bandwidth and the lowpass filter response. Sensor 3 , on the other hand, shows two signal paths received within a short time. Fig. 8 shows the cross-correlation of the two signals of sensor 1 and 3. The two paths add up in the correlation and result in a wider peak with a maximum not at the true time delay. This error can be detected by looking at the peak widths. To estimate the true time delay in such cases, other methods, based on eigenvalue decomposition or maximum likelihood estimation have to be employed.

\section{F. Summary of errors}

The analyzed errors are different in their sources as well as in their effects on the estimation. Each of the observed errors needs to be handled differently. Too low SNR results in random estimates that can not be used for position estimation. The estimates have to be eliminated. Additive noise is the usual expected error and is the basic model for positioning algorithms [6], [5]. It can be additionally compensated by observing longer windows or by averaging over many TDoA estimates before undergoing the localization algorithm. Synchronization errors due to the chosen hardware accuracy can not be compensated without further information, but they can be considered in the system uncertainties. The 


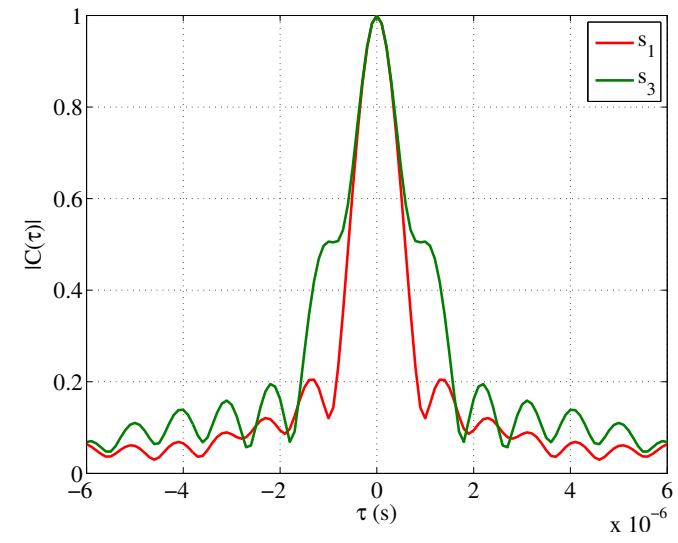

Fig. 7. Autocorrelation of two signals: a signal with one direct path and one with multipath propagation

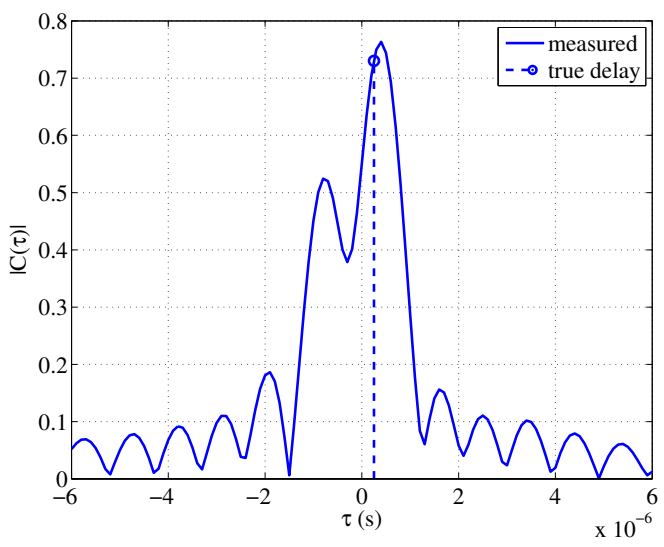

Fig. 8. Cross-correlation of signals with multipath propagation

NLOS error can be mitigated either by eliminating NLOS sensors after identifying them or by weighting different sensor subsets according to their reliability. Multipath propagation can also lead to biased estimates, even with line-of-sight to the transmitter. In multipath scenarios, a different estimation method, for example super resolution methods or maximum likelihood based algorithms offer a good solution.

\section{Algorithms And Positioning Results}

For estimating the position of the transmitter, the Extended Kalman Filter (EKF) was used for both scenarios [11]. The basic equations for the system model are:

$$
\begin{aligned}
x_{k+1} & =\mathbf{A} x_{k}+w_{k} \\
z_{k} & =\mathbf{G} x_{k}+v_{k}
\end{aligned}
$$

whereas $x_{k+1}$ is the state of the system and stands for the position of the transmitter, $\mathbf{A}$ is the transition matrix of the state and $w$ is the system noise, $z_{k}$ is the observation and stands for the estimated TDoAs, $\mathbf{G}$ is the observation matrix that is obtained by linearizing the observation equation and $v_{k}$ is the observation noise. In the static case, the first equation reduces to $x_{k+1}=x_{k}$ so that only the observation



Fig. 9. EKF estimates for the static scenario

noise is modeled and minimized. In the dynamic case, both the movement and the observation noise are modeled.

\section{A. Stationary Scenario}

For chosing a reference sensor, the width of the autocorrelation peak as well as the maximum values of the normalized cross-correlations were measured and the sensor with the highest and the narrowest peaks was chosen as reference sensor.

Fig. 9 shows the position estimates in the stationary scenario. Fig. 10 shows the position error over the filter steps. Three different sets of sensors were chosen. Set 1 includes the 3 TDoA estimates that were not random $\left(\tau_{3,1}, \tau_{4,1}, \tau_{5,1}\right)$. The filter converges fast to a certain position. The remaining bias $(25 \mathrm{~m})$ is a result of sensor 3 having NLOS. The algorithms for NLOS error mitigation both did not work in that case. For the algorithm given in [7], the assumption about higher measurement noise at NLOS sensors was not met here as sensor $3\left(\tau_{3,1}\right)$ produced relatively stable TDoA estimates (see Fig. 6). For the algorithm given in [8], there were not enough LOS subsets to be able to mitigate the error [10]. Both algorithms led to the same or worse results.

Set 2 contains all sensors, including the two sensors that produce random TDoA estimates. The Kalman Filter results show that, eventually, the filter converges to the same position estimate as in set 1 . The only difference is that the filter needs more steps to converge. In the end, the filter practically uses only the same three TDoAs as in set 1 . This result shows how the EKF mitigates measurement noise and how it can produce robust results even with high erroneous TDoAs.

Set 3 includes only LOS sensors. The result shows that the filter converges to the true position whenever the assumption about additive zero mean noise is met, as the NLOS error is not in the calculation anymore.

\section{B. Moving Scenario}

For the moving scenario, a track of 100 seconds, where the transmitter simulated a pedestrian, was chosen. Signals that did not produce a clear correlation peak were eliminated from calculation. Again, a reference sensor was chosen using 




Fig. 10. EKF estimation error for the stationary scenario

the width and the height of the correlation peaks. Here, this procedure was repeated every 5 seconds as the channel conditions are changing.

In this scenario, two time delay estimation methods were used. A simple cross-correlation and interpolation and one maximum likelihood (ML)-based method. The TDoAs estimated by using the cross-correlation method resulted in biased estimates, because the different delayed paths were not resolvable anymore (see Fig. 8).

The ML-based method that was used here was presented in [4] and [12]. Here, we give a short description of the implemented algorithm.

The model of the discrete received signal in multipath can be expressed as:

$$
r_{i}(n)=\sum_{l=1}^{P_{i}} \alpha_{l, i} \cdot s\left(n-\tau_{l, i}\right)+w_{i}(n)
$$

where $n=0, \ldots, K-1$ are the indices of the observed samples, $i=1, \ldots, N$ are the different sensor indices, $P_{i}$ is number of paths for sensor $i, \alpha_{i}$ is the complex factor in each of the multipaths, $\tau_{i}$ is the delay of each path, and $w_{i}$ is a noise term.

There are two assumptions behind the used method:

- The number of received paths $P_{i}$ is known.

- The reference sensor has one path, line-of-sight, to the transmitter $P_{1}=1$. The TDoAs for the different paths in each sensor are $\Delta \tau_{l, i}=\tau_{l, i}-\tau_{1,1}$.

Based on these assumptions, the received signals $r_{k}, \quad k=$ $2, \ldots, N$ can be mathematically described as a function of the received reference signal $r_{1}$.

$$
r_{k}(n)=\sum_{l=1}^{P} \frac{\alpha_{l, k}}{\alpha_{1}} \cdot r_{1}\left(n-\Delta \tau_{l, k}\right)+\tilde{w}(n)
$$

The term $\tilde{w}(n)$ stands for the sum of different noise terms. For each sensor $k$, the ML solution for the TDoA is the vector that minimizes the noise term. The search for the vector that maximizes the likelihood equation was done here by using two principal ideas:



Fig. 11. EKF-estimates for the stationary scenario



Fig. 12. EKF estimation error for the moving scenario

- Pincus' theorm for global maximization [13] to guarantee convergence of the maximum search.

- The importance sampling method as a non iterative technique to calculate the maximum likelihood solution without using a complex multidimensional grid search.

The basic idea of the algorithm can be summed up by the following steps:

- Define a 1-d quasi probability distribution function (pdf) that can be used as a similar pdf for the system. Here, the cross-correlation is used.

- Generate a $\boldsymbol{\Delta} \tau$ vector from the pseudo pdf. Repeat $R$ times to obtain $R$ samples of the vector.

- Weight each of the sampled vectors according to the likelihood function.

- Obtain the final estimate by calculating the circular mean of the weighted samples.

For a more detailed description of the algorithm refer to the original papers.

The results in Fig. 11 show the estimated track and the true one. The results using the described ML-method are more accurate than those of the correlation based method. The correlation method leads to a root mean square error of $25 \mathrm{~m}$ whereas the ML-method leads to a root mean square error of $20 \mathrm{~m}$. 


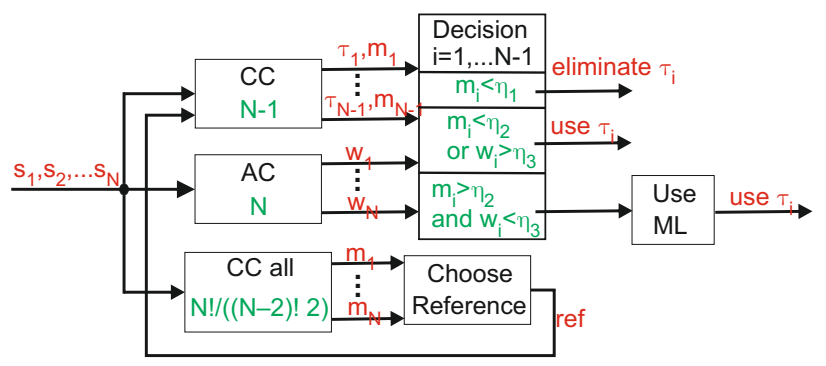

Fig. 13. Intelligent TDoA System

AC: Auto-Correlation, CC: Cross-Correlation, $m_{i}$ :maximum of the normalized correlation, $w_{i}$ : width of the correlation peak

\section{System requirements}

This section presented the localization results considering different aspects of the TDoA errors. A TDoA system that is required for a wide range of applications should be smart. It should be able to identify errors and take action accordingly. In the case of too low SNR, the error can be identified by measuring the normalized cross-correlation maximum. If it goes below a certain threshold, an estimate is labeled as random and the according TDoAs should be eliminated. In case of multipath propagation, the error can be detected by measuring the width of the autocorrelation of the signal. To compensate this error, the mentioned maximum likelihood based algorithm can be applied. For the ML method, defining a good reference sensor is very important to meet the signal model. Last but not least, the biggest challenge and the source of large errors can be undetectable non-line-of-sight. The measured scenario showed a case where the implemented algorithms could not mitigate the NLOS error. Fig. 13 shows the general structure of the required intelligent system. Received and filtered signals $s_{1}, s_{2}, \ldots s_{N}$ should first be tested by calculating the widths $(w)$ of their autocorrelation (AC) as well as the maxima $(m)$ of their cross-correlations (CC). These two features help us first chose the reference sensor. After chosing the reference sensor, only $N-1$ cross-correlations are needed. Their maxima can be compared to thresholds to identify unwanted estimates. Additionally, the maxima and the widths are compared to thresholds to differentiate between multipath and single path scenarios.

\section{CONCLUSION AND Future WORK}

In this paper, the five most important challenges facing passive TDoA systems were presented and analyzed using measured data. The results showed that the TDoA system needs to have the intelligence to identify error sources and cope with them accordingly. The suggested methods, mainly based on the peaks of the autocorrelation and cross-correlation of the signals offer a good approach. The biggest remaining challenge is still non-line-of-sight propagation. The implemented methods failed to detect the error here and it only worked with the a-priori information about the sensor. In future work, the algorithm for the intelligent TDoA system is presented in detail. Other approaches for detecting and mitigating NLOS sensors are also analyzed.

\section{REFERENCES}

[1] C. Knapp and G. Carter, "The generalized correlation method for estimation of time delay," IEEE Transactions on Acoustics, Speech and Signal Processing, vol. 24, no. 4, pp. 320 - 327, Aug. 1976.

[2] F.-X. Ge, D. Shen, Y. Peng, and V.-K. Li, "Super-resolution time delay estimation in multipath environments," IEEE Transactions on Circuits and Systems I: Regular Papers, vol. 54, no. 9, pp. 1977-1986, Sept 2007.

[3] S. Belanger, "Multisensor TDOA estimation in a multipath propagation environment using the EM algorithm," in Conference Record of theTwenty-Ninth Asilomar Conference on Signals, Systems and Computers, 1995, vol. 2, Oct 1995, pp. 1096-1100 vol.2.

[4] A. Masmoudi, F. Bellili, S. Affes, and A. Stephenne, "A non-data-aided maximum likelihood time delay estimator using importance sampling," Signal Processing, IEEE Transactions on, vol. 59, no. 10, pp. 45054515 , Oct 2011

[5] W. Foy, "Position-location solutions by Taylor-series estimation," IEEE Transactions on Aerospace and Electronic Systems, vol. AES-12, no. 2, pp. $187-194$, March 1976.

[6] Y. Chan and K. Ho, "A simple and efficient estimator for hyperbolic location," IEEE Transactions on Signal Processing, vol. 42, no. 8, pp. 1905 -1915, Aug. 1994.

[7] L. Cong and W. Zhuang, "Non-line-of-sight error mitigation in TDOA mobile location," in Global Telecommunications Conference,2001, vol. 1, 2001, pp. $680-684$ vol.1.

[8] P.-C. Chen, "A non-line-of-sight error mitigation algorithm in location estimation," in Wireless Communications and Networking Conference, 1999,1999 , pp. $316-320$ vol.1.

[9] "Ettus research." [Online]. Available: https://www.ettus.com/

[10] N. El Gemayel, S. Meier, and F. Jondral, "On the applicability of the residual weighting algorithm for TDOA," in 4th International Congress on Ultra Modern Telecommunications and Control Systems and Workshops, 2012, Oct 2012, pp. 143-147.

[11] C. Hongyang, D. Ping, X. Yongjun, and L. Xiaowei, "A robust location algorithm with biased extended Kalman filtering of TDOA data for wireless sensor networks," in International Conference on Wireless Communications, Networking and Mobile Computing, 2005, vol. 2, Sept. 2005, pp. $883-886$.

[12] A. Masmoudi, F. Bellili, S. Affes, and A. Stephenne, "A maximum likelihood time delay estimator in a multipath environment using importance sampling," Signal Processing, IEEE Transactions on, vol. 61, no. 1, pp. 182-193, Jan 2013.

[13] M. Pincus, "A closed form solution of certain programming problems," Letter to the Editor, 1968. 